\title{
INTEGRAÇÃO DE DADOS DE SENSORIAMENTO REMOTO MULTI RESOLUÇÕES PARA A REPRESENTAÇÃO DA COBERTURA DA TERRA UTILIZANDO CAMPOS CONTÍNUOS DE VEGETAÇÃO E CLASSIFICAÇÃO POR ÁRVORES DE DECISÃO
}

\author{
Marcelo Lopes Latorre ${ }^{1,2}$, Osmar Abílio de Carvalho Júnior ${ }^{3}$, João Roberto dos Santos ${ }^{1}$ \\ e Yosio Edemir Shimabukuro ${ }^{1}$ \\ Recebido em 29 dezembro, 2006 / Aceito em 7 marco, 2007 \\ Received on December 29, 2006 / Accepted on March 7, 2007
}

\begin{abstract}
This paper aims to develop a methodology of multisensor integration for an Amazon monitoring system. The proposed system employs the Vegetation Continuous Fields (VCF) method that uses the decision tree algorithm. The algorithm uses a set of independent variables, in this case MODIS multi-temporal metrics, to recursively split a dependent variable, in this case training data from class memberships, into subsets, which maximize the reduction of squares of sum of the residuals. The training data are obtained by high-resolution imagery classification (Landsat/TM, ETM+ and CBERS 2/CCD). In this study, an automated algorithm was developed from IDL language in the ENVI software and the statistical procedure of the S-PLUS software. The study area is Mato Grosso State with an extensive area of Amazon forest. The scenes are classified in three classes: forest, non-forest, and water. Comparisons of the final product with regional land cover maps derived from PRODES revel general agreement. Therefore, the results of this study suggest that the methodology is appropriate for land cover determination in the Amazon forest.
\end{abstract}

Keywords: remote sensing, digital image processing, continuous fields, regression trees, Amazon forest.

RESUMO. Este trabalho objetiva desenvolver uma metodologia de integração de multisensores para um sistema de monitoramento da Amazônia. 0 sistema proposto baseia-se no Vegetation Continuous Fields (VCF) que utiliza um algoritmo de árvore de decisão. 0 algoritmo utiliza um conjunto de variáveis independentes, no caso métricas multitemporais do MODIS, para recursivamente particionar uma variável dependente, no caso dados de treinamentos provenientes de classes de uso da terra, em subconjuntos, que maximizam a redução do quadrado da soma residual. Os dados de treinamentos são obtidos pela classificação de imagens de alta resolução (Landsat/TM, ETM+ e CBERS 2/CCD). Neste estudo, um algoritmo foi desenvolvido a partir da linguagem IDL, no programa ENVI, e uma rotina estatística do programa S-PLUS. A área de estudo é o Estado do Mato Grosso com uma extensa área de cobertura de Floresta Amazônica. As cenas são classificadas em três classes: floresta, não floresta e água. Comparações do produto final com o mapa regional de uso da terra derivado do PRODES revelam uma concordância geral. Portanto, os resultados desse estudo sugerem que a metodologia é apropriada para a determinação da cobertura da terra na Floresta Amazônica.

Palavras-chave: sensoriamento remoto, processamento digital de imagens, campos contínuos, árvore de regressão, Floresta Amazônica.

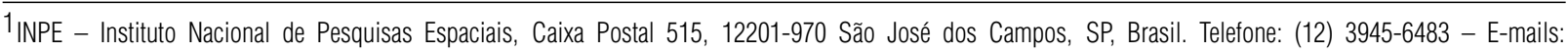
latav@dsr.inpe.br; jroberto@dsr.inpe.br; yosio@dsr.inpe.br

${ }^{2}$ CTA - Comando Geral de Tecnologia da Aeronáutica, Praça Marechal Eduardo Gomes, 50, São José dos Campos, SP, Brasil.

3 Universidade de Brasília, Departamento de Geografia, Campus Universitário Darcy Ribeiro, ICC Norte, 70910-900 Brasília, DF, Brasil. Telefone: (61) 3307-2373; Fax: (61) 3272-1909 - E-mail: osmarjß@unb.br
} 


\section{INTRODUÇÃo}

Nos últimos anos, muito progresso tem sido feito no monitoramento da mudança da cobertura vegetal na Amazônia com dados de satélites. Os primeiros esforços foram realizados com os dados do Landsat/TM (Thematic Mapper) (Shimabukuro \& Almeida Filho, 2002; Espírito-Santo et al., 2005), embora alguns experimentos tenham sido obtidos com outros tipos de sensores como o SPOT/NEGETATION (Carreiras et al., 2002; Carreiras et al., 2006a b) e os Radares de Abertura Sintética (Paradella et al., 1994, 1997, 1998). Embora o imageamento pelos sensores ópticos de resolução espacial média tenha sido usado para mapear e monitorar a Amazônia com certa freqüência e sucesso (PRODES, 2002), sua aplicação vem se mostrando limitada a estudos em uma escala local, devido à dificuldade em obter imagens em série temporal, livres de nuvens, além do alto custo e volume de dados gerados (Foody et al., 1997).

Diante destas limitações, alguns pesquisadores vêm optando pelo emprego de sistemas de mais alta resolução temporal, 0 que permite uma elevada probabilidade de imageamento livre de cobertura de nuvens e, conseqüentemente, um estudo mais apurado da dinâmica de coberturas vegetais (Defries \& Townshend, 1994; Loveland \& Belward, 1997). Entretanto, o principal limitante desse tipo de sistema é a mais baixa resolução espacial, que dificulta a observação local e apresenta pixels provenientes de misturas de classes de interesse.

Como alternativa às dificuldades citadas, tem sido utilizada a integração de dados de multisensores com diferentes resoluções. Para a região da Amazônia destacam-se os sensores de resolução moderada, como o Moderate Resolution Imaging Spectroradiometer (MODIS), e de resolução média, como 0 sensor CBERS 2/CCD (Charge Couple Device). Futuramente, poderá ainda dispor do sensor aerotransportado de Varredura Hiperespectral (HSS) do Sistema de Vigilância da Amazônia (SIVAM).

Deste modo, o presente trabalho possui como objetivo desenvolver uma metodologia para o monitoramento da dinâmica da cobertura da terra na Amazônia, enfatizando a integração de dados de diferentes sensores e resoluções (Terra/MODIS, Landsat5/TM, Landsat7/ETM+ e CBERS 2/CCD). 0 modelo utilizado é 0 de Campos Contínuos de Vegetação (Vegetation Continuous Fields - VCF) desenvolvido por Hansen et al. $(2002,2003)$. 0 procedimento é automatizado e gera uma árvore de regressão (Venables \& Ripley, 1994) para as áreas de treinamento entre os dados do sensor Landsat/TM, Landsat7/ETM+ ou IKONOS com relação aos dados do sensor MODIS (Hansen et al., 2000, 2002). Espera-se que esta metodologia auxilie e complemente, em especial, o projeto SIVAM na sua missão de monitoramento, e também, a projetos já consagrados e desenvolvidos para a Amazônia Brasileira, como é 0 caso do Projeto de Estimativas de Desflorestamento da Amazônia, PRODES (PRODES, 2002).

\section{Sensor MODIS}

0 comitê espacial norte americano mantém um programa de longa duração de pesquisas de observação da superfície terrestre, oceanos e atmosfera, o sistema "Earth Observing System" (EOS), financiado pelo programa da NASA "Earth Science Enterprises" (ESE). 0 objetivo principal da ESE é determinar como a Terra está mudando e quais as consequêencias para a vida neste planeta, desenvolvendo um entendimento de seu funcionamento como um sistema único e interligado (Justice et al., 2002).

0 principal instrumento do projeto EOS é o sensor MODIS que apresenta entre suas características (Barker et al., 1992): (a) uma ampla cobertura espacial e espectral (Tabela 1) e (b) consiste na primeira ferramenta dos satélites EOS na condução das pesquisas de mudanças globais. As trinta e seis bandas espectrais deste sensor estão localizadas em função de um comprimento de onda definido para a observação de feições das propriedades das nuvens e sua dinâmica, as propriedades da vegetação na cobertura terrestre e a temperatura da superfície dos oceanos no mundo (Salomonson \& Toll, 1990). Desta forma, o MODIS permite aperfeiçoar os estudos realizados com o Advanced Very High Resolution Radiometer (AVHRR), uma vez que, possui a capacidade de rastrear um amplo conjunto de dados da Terra (Strahler et al., 1999).

\section{Árvore de decisão}

Uma árvore de decisão tem a função de dividir um conjunto de treinamento, até que cada subconjunto obtido deste particionamento contenha casos de uma única classe. Uma árvore é composta pelas seguintes unidades: (a) um nó raiz formado a partir de todos os dados, (b) um conjunto hierárquico de nós internos denominados partições ("Split") responsáveis pela tomada de decisão, utilizando teste lógico, que define o próximo nó descendente ou filho, e (c) nós terminais chamados de folhas ("leaves") caracterizados por não possuírem nó descendente. A árvore de decisão binária divide cada nó intermediário em dois nós descendentes: 0 nó esquerdo (quando os dados satisfazem 0 teste lógico) e o nó direito (quando não satisfazem). A divisão em cada nó interno de uma árvore é definida por critérios estatísticos sobre os dados de treinamento. No método de árvore de regressão o processo decisório em cada nó terminal ou folha contém uma constante (normalmente, a média) ou uma equação linear referente a um determinado conjunto de dados. 
Tabela 1 - Especificações técnicas do sensor MODIS. Adaptada de Justice et al. (2002).

\begin{tabular}{|c|c|}
\hline Órbita & $\begin{array}{l}705 \text { km, síncrona com o Sol, polar; 10:30 a.m. descendente; } \\
\text { 1:30 p.m. ascendente }\end{array}$ \\
\hline Alcance Espectral & $0,4-14,4 \mu \mathrm{m}$ \\
\hline Cobertura Espectral & $\begin{array}{l} \pm 55^{\circ}, 2330 \mathrm{~km} \text { em fileira } \\
\text { ("scans" contínuos em nadir no equador) }\end{array}$ \\
\hline Resolução Espacial & $\begin{array}{l}250 \text { m ( } 2 \text { bandas), } 500 \text { m ( } 5 \text { bandas), } \\
1000 \text { m ( } 29 \text { bandas) em nadir }\end{array}$ \\
\hline Precisão Radiométrica & $\begin{array}{l}5 \% \text { Absoluto, }<3 \mu \mathrm{m} ; 1 \% \text { Absoluto, } \\
\quad>3 \mu \mathrm{m} ; 2 \% \text { de reflectância }\end{array}$ \\
\hline Cobertura de repetição & $\begin{array}{c}\text { Diária, a norte da latitude } 30^{\circ} \text { e a cada dois dias, } \\
\text { para latitudes inferiores a } 30^{\circ}\end{array}$ \\
\hline Quantização & 12 bits \\
\hline Taxa de Dados & 6,2 Mbps (média), 10,8 Mbps (dia), 2,5 Mbps (noite) \\
\hline Força & 162,5 W (média para uma órbita), 168,5 W (pico) \\
\hline
\end{tabular}

Inúmeras técnicas de construção de árvores têm sido desenvolvidas nos últimos trinta anos, sendo, o algoritmo ID3 um dos primeiros algoritmos de árvore de decisão baseado em sistemas de inferência e conceitos de aprendizagem (Quinlan, 1986). Logo após, foram elaborados diversos algoritmos, sendo os mais conhecidos: CART ("Classification and Regression Tree") e C4.5/5.0 (Quinlan, 1993; Friedl et al., 1999). Para este trabalho foi empregado 0 método de árvore de regressão do programa estatístico S-PLUS, em função de sua flexibilidade, simplicidade intuitiva e eficiência computacional. Este programa emprega o conjunto de variáveis independentes (métricas) para recursivamente dividir uma variável dependente (dados de treinamentos obtidos das cenas TM, ETM+ e CBERS 2/CCD), em subconjuntos, os quais maximizam a redução na soma dos quadrados residuais.

Um importante passo na estimação de uma árvore está em corrigi-la de uma possível superestimação, através do processo de "poda". Convencionalmente, uma árvore é crescente até que todas as observações de treinamento sejam corretamente classificadas. 0 algoritmo utilizado define duas amostras a partir dos dados de treinamento, 0 qual uma amostra é usada para desenvolver (GTR) a árvore e a outra amostra para "podá-la" (PTR), este processo é denominado de resubstituição. 0 resultado final é a obtenção de uma estrutura em árvore com divisões hierárquicas que permitem o estabelecimento das classes (Figura 1). Os resultados obtidos são organizados de maneira compacta, podendo ser utilizado para classificar novos casos similares (Friedl \& Brodley, 1997).

\section{Área de estudo}

A área de estudo consiste no Estado do Mato Grosso que apresenta uma área territorial de cerca de $900.000 \mathrm{~km}^{2}$ (Figura 2). 0 Mato Grosso caracteriza-se por apresentar uma grande diversidade de tipos de formações vegetais, além de estar sujeita a uma alta taxa na conversão da sua cobertura vegetal, devido à utilização da agricultura mecanizada e pecuária nas áreas de cerrado, bem como o desmatamento e queimadas nas áreas de floresta.

Este estado não sofre interferência das influências marítimas, permitindo que a variação da latitude seja responsável pela temperatura de cerca de $26^{\circ} \mathrm{C}$ no extremo norte e de $22^{\circ} \mathrm{C}$ no extremo sul da região. 0 relevo, através da altitude, faz com que as altas chapadas sedimentares e superfícies cristalinas do centro-sul da região possuam temperatura média anual entre $22^{\circ} \mathrm{C}$ e $20^{\circ} \mathrm{C}$. Em razão da pouca significância da topografia sobre a pluviosidade, a quantidade média da precipitação durante 0 ano sobre o território apresenta um padrão de distribuição característico. Distingue-se um núcleo mais chuvoso ao norte do Mato Grosso, onde a pluviosidade anual atinge valores elevados, superiores a $2.700 \mathrm{~mm}$, com estes valores decrescendo para 0 leste e sul atingindo $1.250 \mathrm{~mm}$. Entretanto, essas precipitações não se distribuem igualmente através do ano. Seu regime é caracteristicamente tropical, com máxima no verão e mínima no inverno. Mais de 70\% da precipitação anual ocorrem de novembro a março, sendo geralmente mais chuvoso o trimestre janeiro-março no norte do Estado, dezembro-fevereiro no centro e novembro- 


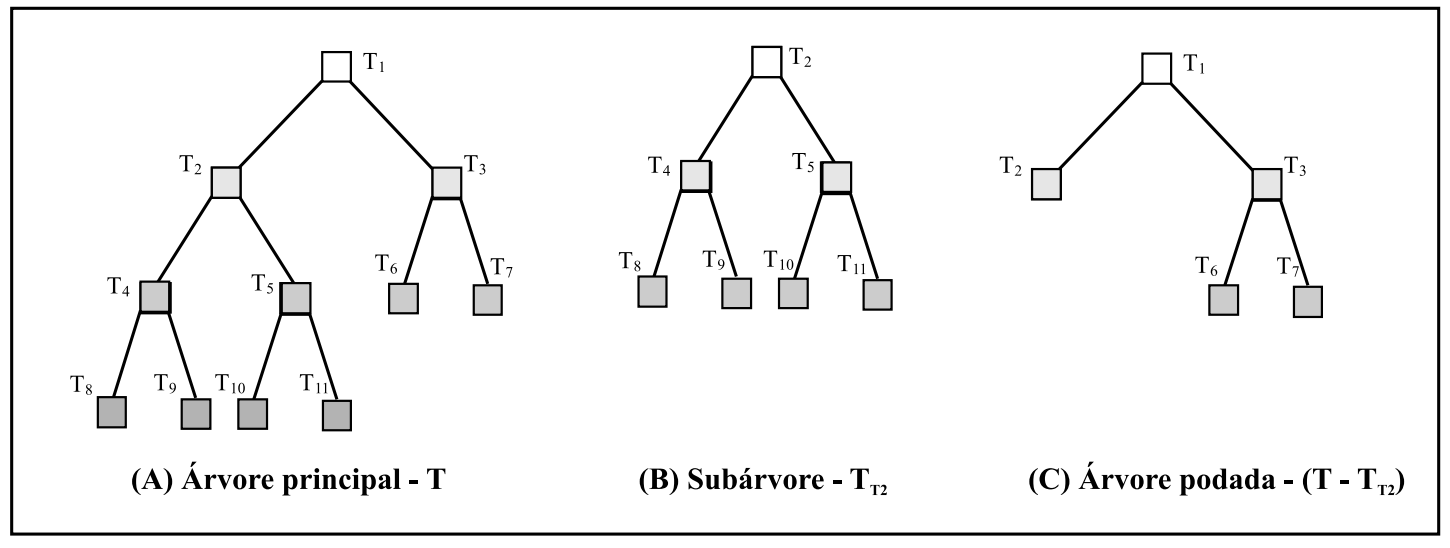

Figura 1 - Exemplo de poda. (A) árvore completa; (B) subárvore; e (C) árvore final após a poda. (Modificado de Breiman et al., 1984).

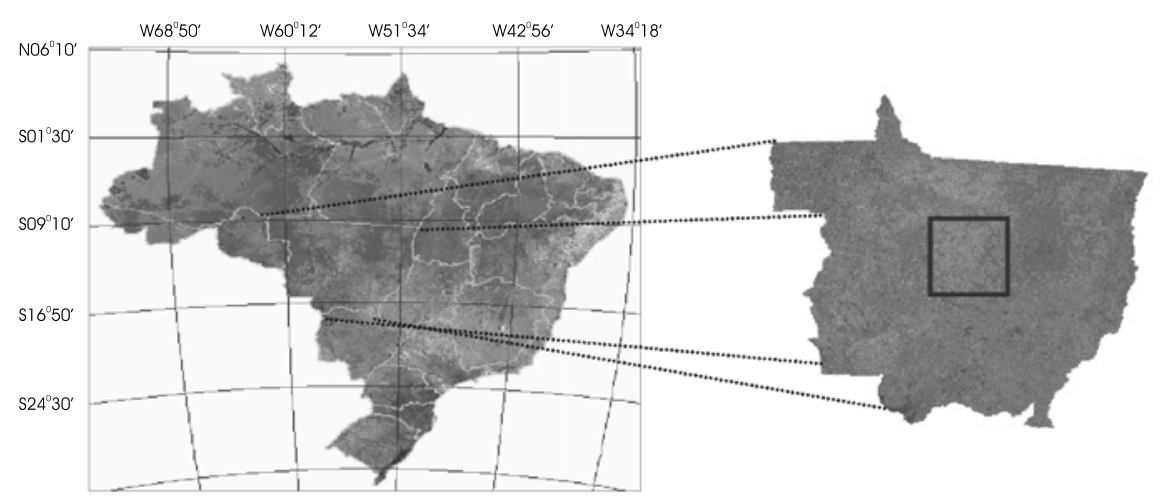

Figura 2 - Localização do Estado do Mato Grosso salientando a área exemplificada no presente trabalho (Fonte: Adaptada de Anderson et al., 2005).

janeiro no sul. Durante esses trimestres chove em média 45 a $55 \%$ do total anual. Em contrapartida, o inverno é excessivamente seco. Nessa época do ano as chuvas são muito raras, ocorrendo em média, de 4 a 5 dias chuvosos por mês (Nimer, 1977).

0 relevo nesta região tem como substrato a porção do escudo brasileiro denominada de Maciço Mato-Grossense (Moreira, 1977). Este é limitado ao norte pelas Bacias Amazônicas, a leste pela Bacia do São Francisco, a oeste pela borda oriental andina e ao sul-sudeste, pela Bacia de sedimentação do Paraná.

\section{MATERIAIS E MÉTODOS}

\section{Imagens e programas utilizados}

Para a realização deste estudo foram utilizadas imagens dos seguintes sensores: (a) Landsat5/TM - banda azul (450-520 nm), verde $(520-600 \mathrm{~nm})$, vermelho $(630-690 \mathrm{~nm})$ e infravermelho próximo (760-900 nm), referente a duas cenas (225-69 e 22668) obtidas nos meses de agosto e setembro de 2002, res- pectivamente; (b) Landsat7/ETM+ - banda azul (450-520 nm), verde (530-610 nm), vermelho $(630-690 \mathrm{~nm})$ e infravermelho próximo (780-900 nm), referente a três cenas obtidas nos meses de setembro (225-68, 226-69) e outubro (226-71) de 2002; (c) CBERS 2/CCD - banda azul ( $450-520 \mathrm{~nm})$, verde $(520-590 \mathrm{~nm})$, vermelho (630-690 nm) e infravermelho próximo (770-890 nm) referente a uma cena obtida (166-116) no mês de novembro de 2003; (d) MODIS $500 \mathrm{~m}$ - bandas: azul (459-479 nm), verde (545-565 nm), vermelho (620-670 nm), infravermelho próximo (841-876 nm) e infravermelho médio (1230-1250 nm, 1628$1652 \mathrm{~nm}$ e 2105-2155 nm), referentes às composições de 32 dias do ano de 2002. Uma composição de imagens MODIS é formada considerando para cada célula os valores mínimos dentre as imagens diárias no período de trinta e dois dias, com 0 objetivo de reduzir a presença de nuvens. 0 emprego de diversos sensores teve como objetivo integrar e avaliar dados de diferentes resoluções (radiométricas, espaciais e espectrais) no desenvolvimento da metodologia proposta. 
Os programas utilizados foram: (a) programa ENVI 3.6 (ENvironment for Visualizing Images) para o processamento das imagens (ENVI, 2002); (b) linguagem IDL (Interactive Data Language) 5.6 para 0 desenvolvimento do programa de monitoramento e a sua interface; (c) programa S-PLUS (Kaluzny et al., 1998) empregado no treinamento das variáveis (dependentes e independentes) criadas, através de um algoritmo de regressão baseado em árvore, e aplicadas no modelo proposto; (d) programa PCl Geomatica 9.1 para obter os pontos para o registro das imagens de melhor resolução (TM, ETM+ e CBERS 2/CCD) com os dados MODIS (500 m).

\section{Desenvolvimento do sistema de monitoramento}

0 sistema proposto para o monitoramento da Amazônia Legal, baseia-se no método VCF (Defries et al., 1997; Defries \& Chan, 2000; Hansen et al., 2002, 2003) com as seguintes adequações: (a) maior automatização na geração do produto em relação aos já existentes para a Amazônia Brasileira; (b) criação de um sistema aplicando as técnicas de campos contínuos, mas de maneira mais simplificada (menor número de classes), que permita, futuramente, gerar produtos de intervalos menores, como exemplo, quadrimestral (como os dados de $500 \mathrm{~m}$ do MODIS); (c) utilização de novos sensores como o CBERS 2/CCD; (d) facilidade na interação com o modelo desenvolvido, uma vez que cerca de $90 \%$ de todo o processo é realizado com um único programa, neste caso o ENVI; (e) utilização do processamento de dados em linguagem IDL; e (f) criação de uma interface gráfica para uma maior interação junto ao usuário.

Desta forma, 0 desenvolvimento do sistema de monitoramento apresenta as seguintes fases: (a) criação das variáveis independentes (as métricas - a partir dos dados MODIS $500 \mathrm{~m}$ ); (b) criação das variáveis dependentes (dados de treinamentos gerados a partir de cenas de melhor resolução espacial: ETM+, TM e CBERS 2/CCD); (c) utilização da regressão em árvore do programa S-PLUS e emprego na cena MODIS; e (d) criação da interface gráfica para uma maior interação junto ao usuário.

\section{Obtenção das métricas}

Diversos tipos de métricas multitemporais para a descrição da fenologia da vegetação têm sido sugeridos. Muitos autores têm obtido métricas ligadas a eventos fenológicos, baseados em uma série temporal de dados NDVI, incluindo taxa de senescência, tempo de pico de NDVI, máximo NDVI, entre outros (Reed et al., 1994).

Neste trabalho, as bandas da imagem MODIS $500 \mathrm{~m}$ relativas à cobertura terrestre (azul - 459-479 nm, verde - 545-565 nm, vermelho - 620-670 nm, infravermelho próximo - 841-876 nm, e médio - 1230-1250 nm, 1628-1652 nm e 2105-2155 nm) foram transformadas em métricas anuais e de NDVI, as quais foram usadas como dados de entrada (variáveis independentes) para se gerar a classificação.

A Figura 3 exemplifica a obtenção de métricas para a banda do vermelho relativa ao dado MODIS de $500 \mathrm{~m}$ (anual). Neste caso, considerando apenas as 11 composições referentes ao canal do vermelho, realiza um ordenamento decrescente pixe/ a pixe/ em função do seu valor espectral. Este ordenamento irá gerar ao final onze novas composições relativas à banda do vermelho, variando do maior ( $1^{\mathrm{a}}$ composição) para 0 menor valor ( $11^{\text {a }}$ composição). Uma vez estabelecido 0 ordenamento, dáse início ao cálculo das métricas (médias, amplitudes, mínimas, máximas, entre outros). Este processo é aplicado, também, aos demais canais.

\section{Dados de treinamento}

Como dados de treinamento pode-se utilizar tanto parte da imagem ou a imagem inteira. Os dados de treinamento no presente trabalho são obtidos pelos seguintes passos: (a) classificação supervisionada da imagem de melhor resolução (CBERS 2/CCD, Landsat5/TM e Landsat7/ETM+), neste caso, pelo método de Máxima Verossimilhança (Maxver); (b) atribuição de valores de porcentagem de cobertura vegetal para as classes da imagem classificada pelo Maxver; e (c) conversão e agregação da imagem de porcentagem de cobertura para a resolução do MODIS (500 m) utilizando a média (Figura 4). Neste trabalho, como a ênfase é 0 desenvolvimento metodológico, optou-se por definir apenas três classes na classificação pelo método Maxver: floresta, não floresta e água/áreas inundáveis. A conversão da imagem para a resolução do MODIS permite uma mesma resolução espacial para as métricas e os dados de treinamento.

0 registro dos dados de treinamento de acordo com os dados MODIS foi realizado pelos programas ENVI (programa em IDL criado especialmente para esta tarefa) e o PCI 9.1 Geomatica. 0 programa PCI foi utilizado apenas para coletar os pontos de controle necessários ao registro das cenas. Em seguida, estes pontos são inseridos no ENVI para a realização do registro.

As amostras selecionadas e suas informações são convertidas em arquivos no formato de texto (.TXT), que são inseridos no programa S-PLUS para o treinamento da árvore de regressão. Cabe salientar que, não foi realizada nenhuma integração entre os programas ENVI/IDL e $S$-PLUS, sendo suas aplicações realizadas independentemente. 


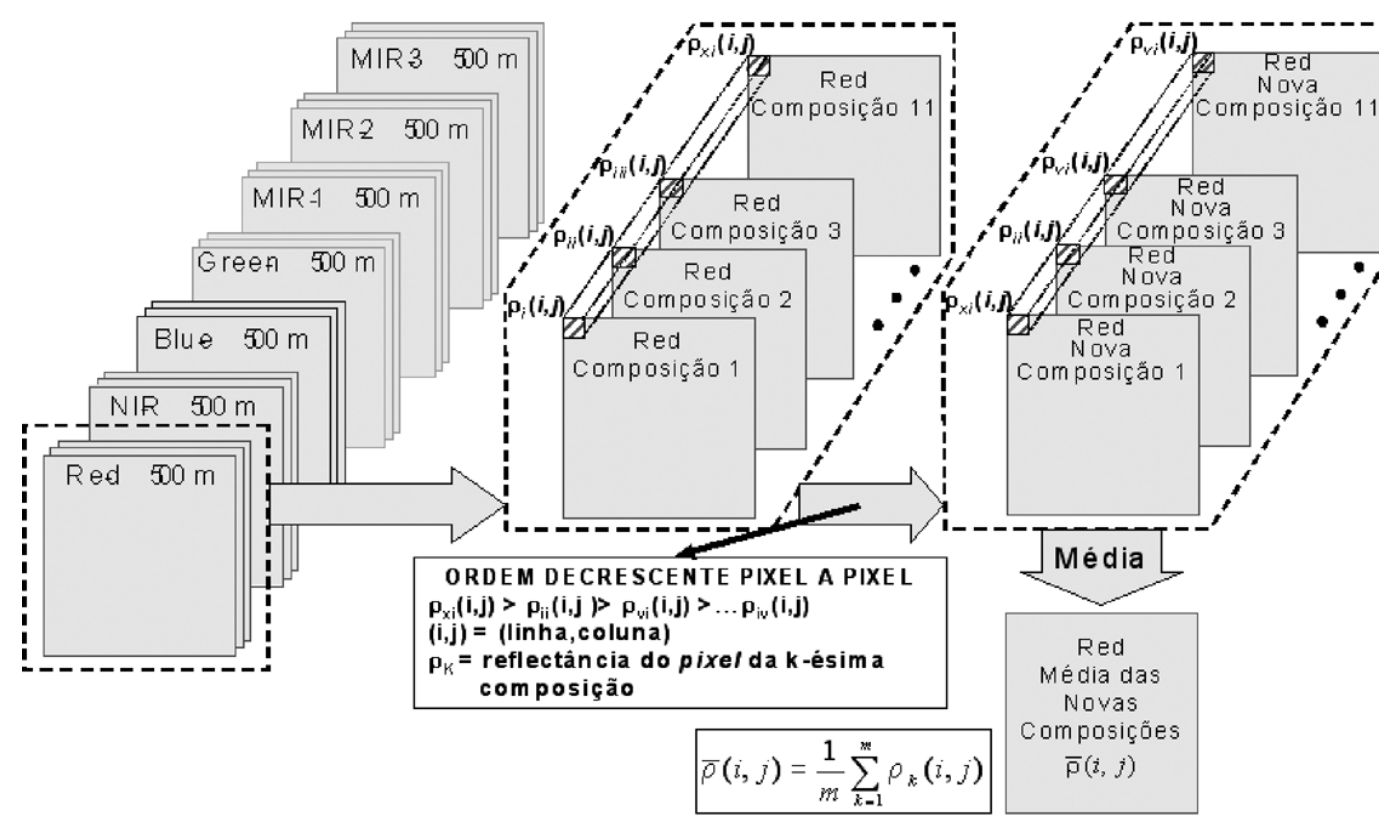

Figura 3 - Exemplo na obtenção de métricas para o canal vermelho do dado MODIS $500 \mathrm{~m}$ (anual).
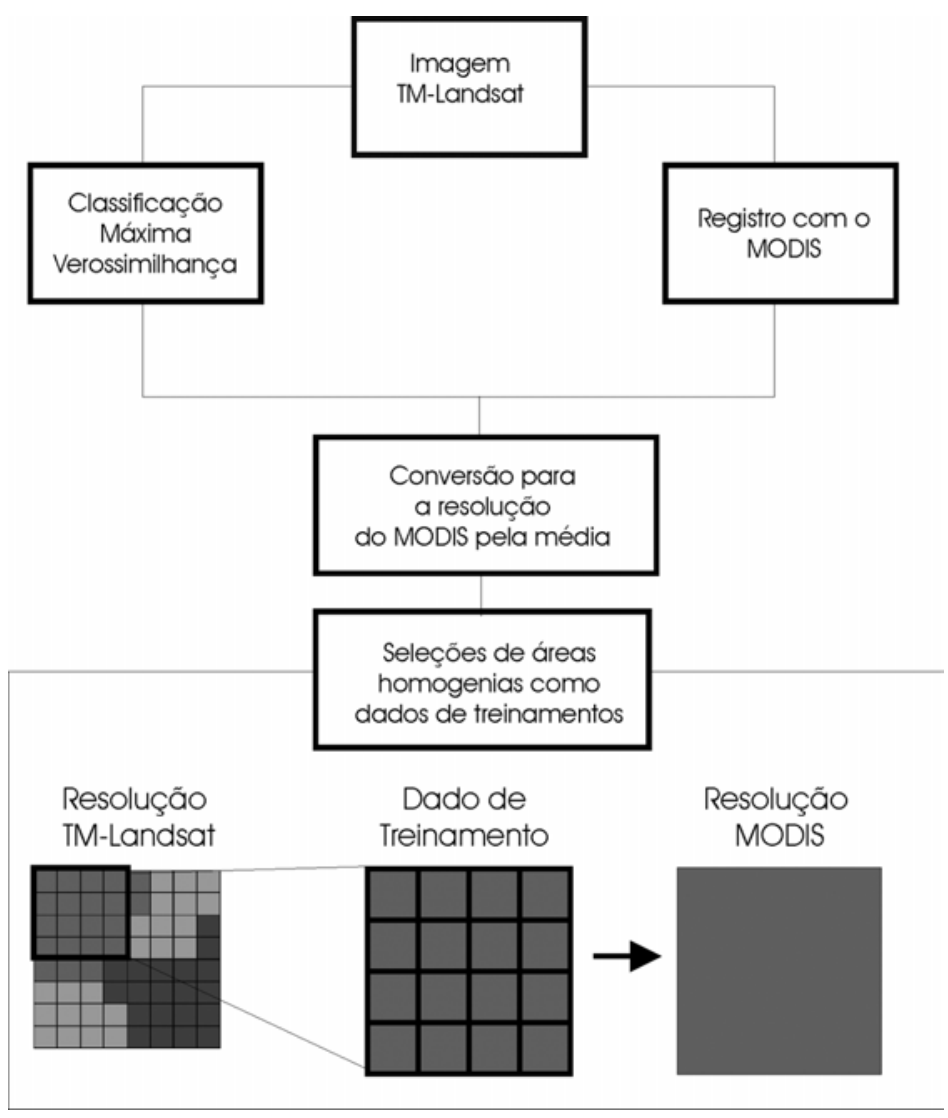

Figura 4 - Etapas metodológicas para a obtenção de um dado de treinamento. 


\section{Geração e comparação dos resultados da regressão em árvore}

Nesta fase, os resultados obtidos pela árvore de regressão são convertidos em linguagem IDL e aplicados à cena do MODIS, para a realização da classificação. No presente estudo são gerados e comparados modelos considerando apenas um tipo de dado de treinamento (produto de um único sistema sensor - Landsat $7 /$ ETM+, Landsat 5/TM ou CBERS 2-CCD), como também, o emprego conjunto de dois ou mais tipos de dados (procedentes de sistemas sensores distintos - Landsat 5/TM, Landsat 7/ETM+e CBERS 2 -CCD). A cena MODIS é classificada em três classes: (a) floresta, (b) não floresta e (c) água/áreas inundáveis. Os meIhores resultados são separados por intermédio de interpretação visual, complementada por uma análise da matriz de confusão acurácia global e coeficiente Kappa (Tabela 2).

Tabela 2 - Classificação em função do coeficiente Kappa.

\begin{tabular}{|c|c|}
\hline Kappa & Conceito \\
\hline$<0$ & Péssima \\
\hline $0<k \leq 0.2$ & Má \\
\hline $0.2<k \leq 0.4$ & Razoável \\
\hline $0.4<k \leq 0.6$ & Boa \\
\hline $0.6<k \leq 0.8$ & Muito Boa \\
\hline $0.8<k \leq 1.0$ & Excelente \\
\hline
\end{tabular}

0 melhor resultado obtido com a metodologia proposta é comparado com os dados provenientes do Projeto PRODES. Essa comparação teve como objetivo avaliar a eficiência do método, bem como identificar a sua viabilidade no monitoramento da cobertura terrestre da Amazônia. Com o propósito de comparar com o Projeto PRODES foram estabelecidas algumas adaptações, uma vez que, os produtos apresentavam projeções, resoluções e metodologias diferenciadas: (a) os mapas foram colocados na mesma projeção, utilizando como referência os dados MODIS; (b) os dados do PRODES passaram para a mesma resolução espacial do MODIS de 467,563 m, uma vez que os dados PRODES estavam com resolução de 120 m; (c) estabelecimento das mesmas classes; e (d) as cenas foram recortadas de maneira correspondente, ou seja, na mesma grade ou área de atuação. Devido o PRODES não ter sido realizado na área do Pantanal, essa área foi desconsiderada na comparação.

\section{RESULTADOS E DISCUSSÃO}

0 programa desenvolvido estabelece uma interface de fácil interação com o usuário. Desta forma, tanto o desencadeamento das diferentes etapas, bem como sua interação permitiu um ganho considerável na automação do processo metodológico e, sobretudo no tempo de processamento (aproximadamente duas horas) (Figura 5).

A Figura 6 apresenta a classificação obtida ao se empregar apenas um tipo de dado de treinamento, proveniente do sensor Landsat 7 (ETM+ 226-69). Verifica-se que neste caso conseguiuse um nível de detalhamento bem satisfatório para o resultado final, levando em consideração o número reduzido de classes. 0 valor da acurácia global é de 84,04\% e o coeficiente Kappa é de 0,7610 .

A área do Pantanal (quadrante inferior esquerdo da cena MODIS) é a maior responsável pela porcentagem de erro. Acredita-se que esse resultado tenha sido pela falta de qualidade dos dados de treinamento para a região em questão, provavelmente, devido à alta sazonalidade e complexidade (Fisionômica/Estrutural) desta área contribuíram, também, para os resultados obtidos.

Um resultado obtido ao se empregar dois ou mais tipos de dados de treinamentos (Landsat 7/ETM+ e TM-Landsat 5) conjuntamente é demonstrado na Figura 7. De uma maneira geral, observou-se que as diferenças não foram significativas, como demonstram os dados de acurácia global $(81,85 \%)$, coeficiente kappa $(0,6815)$ e a análise visual. Novamente, a região do Pantanal é a porção da imagem com maior dificuldade de classificação.

Através das análises comparativas verifica-se que as diferenças, tanto visuais, quanto quantitativas, não foram muito significativas, mostrando que para a situação do emprego de apenas um único tipo de dado é suficiente para se gerar mapas satisfatórios.

A comparação do modelo proposto usando apenas um dado de treinamento (Landsat 7/ETM+ 226-69) com o PRODES apresenta alta correspondência visual e valores satisfatórios de Acurácia Global (87,48\%) e Coeficiente Kappa (0,7437) (Figura 8). Acredita-se que estes resultados tenham sido atingidos, tendo em vista 0 fato da área correspondente ao Pantanal não ter sido incluída na análise comparativa, como comentado anteriormente.

\section{CONCLUSÕES E SUGESTÕES}

Com relação ao modelo proposto para o monitoramento da cobertura terrestre pode-se concluir que: (1) é viável para a região amazônica, tendo em vista os resultados comparativos com os do Projeto PRODES; (2) permite o emprego de diversos tipos de 


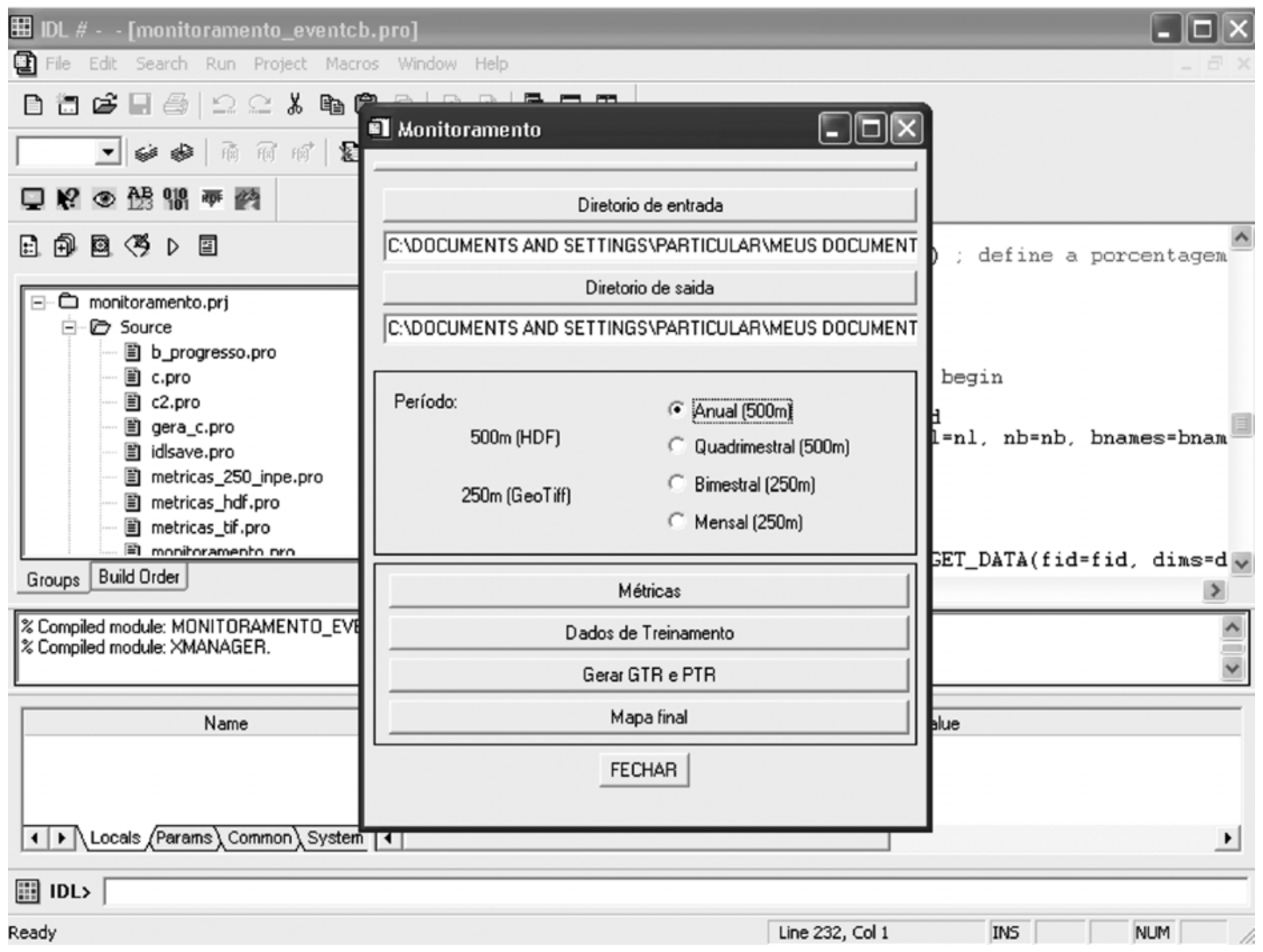

Figura 5 - Interface criada para a aplicação do sistema de monitoramento.

dados de treinamento, tanto isoladamente, quanto em conjunto; e (3) mostrou-se viável ao se aplicar apenas um dado de treinamento na obtenção do mapa final dos dados MODIS conseguindo com isto, uma redução considerável no tempo de processamento dos dados.

Como forma de dar prosseguimento ao estudo realizado pode-se apresentar como sugestões: (1) criar novas métricas ou variáveis, implementá-las e testá-las para verificar a sua viabilidade no modelo proposto; (2) verificar a viabilidade do emprego dos novos produtos (cenas de alta resolução) na metodologia apresentada, como os gerados, futuramente, pelo SIVAM: HSS, MSS, entre outros; (3) ampliar ou aperfeiçoar a interface criada em função das novas necessidades e objetivos a serem criados pelo usuário final; (4) verificar a viabilidade do modelo proposto para áreas de intensa degradação ambiental empregandose dados MODIS de $250 \mathrm{~m}$; e (5) verificar a possibilidade de migração deste processo metodológico para a aplicação em programas livres.
Tendo em vista à necessidade de aplicação de modelos de monitoramento para as regiões tropicais, como o Brasil, a presente abordagem metodológica permite uma otimização no tratamento dos dados. Espera-se, que este tipo de produto gerado venha a contribuir para a função da Força Aérea junto à Amazônia Brasileira, complementando, ainda, o trabalho desenvolvido pelo INPE através do PRODES.

\section{AGRADECIMENTOS}

Os autores agradecem o apoio recebido durante a realização deste trabalho das seguintes instituições: Conselho Nacional de Desenvolvimento Científico e Tecnológico (CNPq) pelo financiamento de bolsas de pesquisa; Instituto Nacional de Pesquisas Espaciais (INPE) e Comando-Geral de Tecnologia Aeroespacial (CTA). Agradecemos ao pesquisador M.C. Hansen e aos demais pesquisadores da Universidade de Maryland pelo apoio e auxílio científico prestado e aos revisores da Revista Brasileira de Geofísica pelas sugestões feitas no texto. 

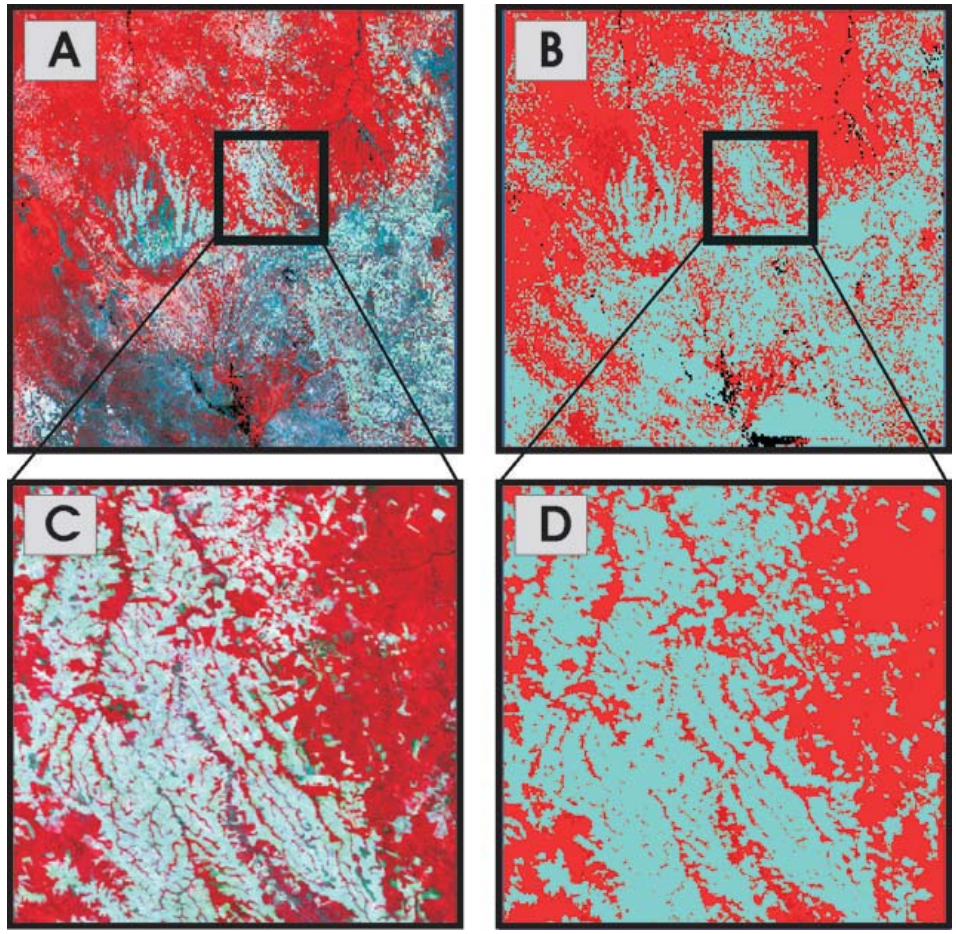

Figura 6 - Comparações entre as imagens MODIS (500 m) da cena H12V10 do Estado do Mato Grosso e a imagem classificada utilizando apenas um tipo de dado de treinamento (ETM+ 226-69): (a) composição colorida da imagem MODIS - R(NIR), G(RED) e B(BLUE); (b) imagem classificada pelo modelo proposto, considerando três classes - não floresta (ciano), floresta (vermelho) e água/áreas inundáveis (preto), (c) detalhe da imagem MODIS e (d) detalhe da imagem classificada.

\section{REFERÊNCIAS}

ANDERSON LO, SHIMABUKURO YE, LATORRE ML, ESPÍRITO-SANTO FDB, LIMA A, DUARTE V, ARAI E, DEFRIES RS, MORTON D, HANSEN M \& JASINSKY E. 2005. Coleta de dados para estudos de cobertura arbórea, mapeamento da cobertura da terra, desmatamento e avaliação de unidades de conservação/territórios indígenas: metodologias de trabalhos de campo. São José dos Campos: INPE, (INPE-12304-NTC/368). $36 \mathrm{pp}$.

BARKER JL, HARDEN MK, ANUTA EA, SMID J \& HOUGT D. 1992. MODIS spectral sensivity study: requirements and characterization. Washington: Nasa, 84 pp.

BREIMAN L, FRIEDMAN JH, OLSHEN RA \& STONE CJ. 1984. Classification and regression trees. Belmont, CA: Wadsworth International, $358 \mathrm{pp}$.

CARREIRAS JMB, PEREIRA JMC \& SHIMABUKURO YE. 2002. Fraction images derived from SPOT-4 VEGETATION data to assess land-cover change over the State of Mato Grosso, Brazil. International Journal of Remote Sensing, 23: 4979-4983.

CARREIRAS JMB, PEREIRA JMC \& SHIMABUKURO YE. 2006a. Land cover mapping in the Brazilian Amazon using SPOT-4 VEGETATION data and machine learning classification methods. Photogrammetric Engineering and Remote Sensing, 72(8): 897-910.

CARREIRAS JMB, PEREIRA JMC, CAMPAGNOLO ML \& SHIMABUKURO YE. 2006b. Assessing the extent of agriculture/pasture and secondary succession forest in the Brazilian Legal Amazon using SPOT VEGETATION data. Remote Sensing of Environment, 101: 283-298.

DEFRIES RS \& TOWNSHEND JRG. 1994. NDVI - derived land cover classification at global scales. International Journal of Remote Sensing, 15: 3567-3586.

DEFRIES RS, HANSEN MC, STEININGER M, DERLAYAH R, SOHLBERG R \& TOWNSHEND J. 1997. Subpixel forest cover in Central Africa for multisensor, Multitemporal data. Remote Sensing of Environment, 60: 228-246.

DEFRIES RS \& CHAN JC-W. 2000. Multiple criteria for evaluating machine learning algorithms for land cover classification from satellite data. Remote Sensing of Environment, 74: 503-515.

ENVI. ENvironment for Visualizing Images. 2002. User's Guide. Lafayette, Colorado, USA: Better Solutions Consulting Limited Liability Company. 614 pp. 


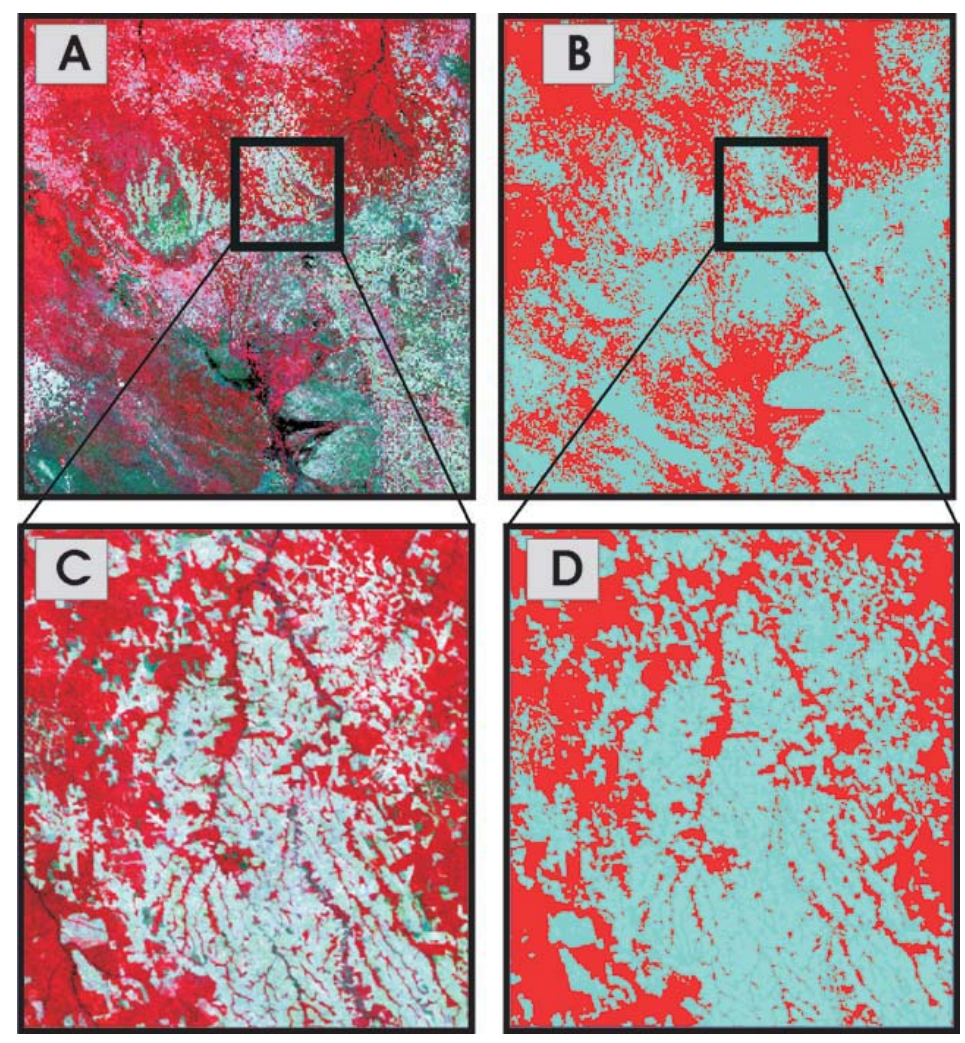

Figura 7 - Comparações entre as imagens MODIS (500 m) da cena H12V10 do Estado do Mato Grosso e a imagem classificada utilizando conjuntamente dois tipos de dados de treinamentos (ETM+ 226-69 e TM 225-68). (a) composição colorida da imagem MODIS - R(NIR), G(RED) e B(BLUE); (b) imagem classificada pelo modelo proposto, considerando três classes - não floresta (ciano), floresta (vermelho) e água/áreas inundáveis (preto), (c) detalhe da imagem MODIS e (d) detalhe da imagem classificada.
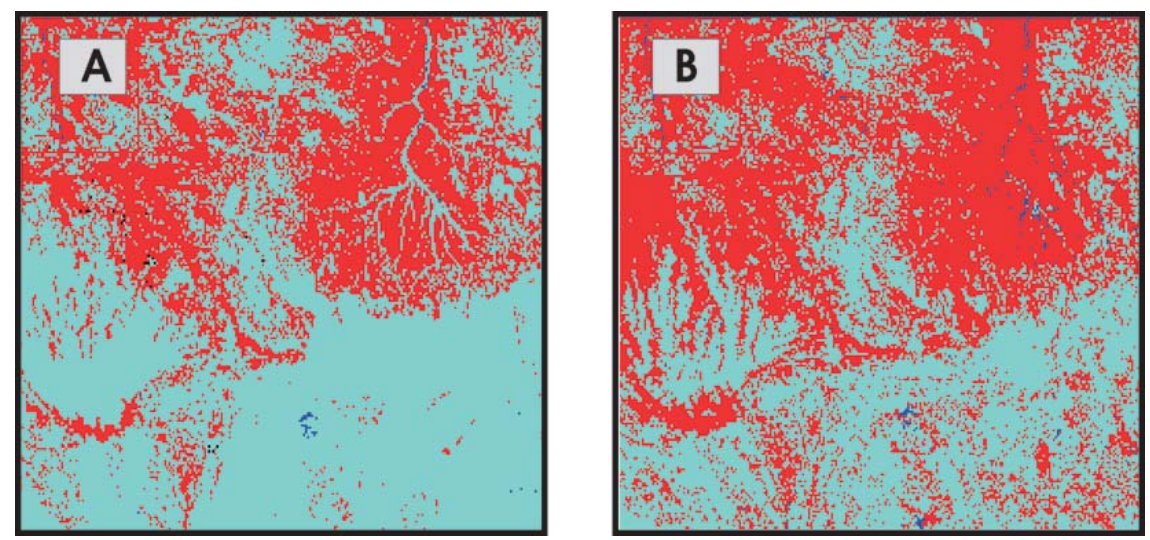

Figura 8 - Comparação entre: (a) dados do Projeto PRODES (área do Estado do Mato Grosso - ano de 2002) e (b) modelo proposto (empregando um único dado de treinamento - Landsat 7/ETM+ 226-69). 
ESPÍRITO-SANTO FDB, SHIMABUKURO YE \& KUPLICH TM. 2005. Mapping forest successional stages following deforestation in Brazilian Amazonia using multi-temporal Landsat images. International Journal of Remote Sensing, 26(3): 635-642.

FOODY GM, LUCAS RM, CURRAN PJ \& HONZAK M. 1997. Nonlinear mixture modelling without end-members using an artificial neural network. International Journal of Remote Sensing, 18(4): 937-953.

FRIEDL MA \& BRODLEY CE. 1997. Decision tree classification of land cover from remotely sensed data. Remote Sensing of Environment, 6: 399-409.

FRIEDL MA, BRODLEY CE \& STRALHER AH. 1999. Maximizing land cover classification accuracies produced by decision trees at continental to global scales. IEEE Transactions on Geoscience and Remote Sensing, 37(2): 969-977.

HANSEN MC, DEFRIES RS, TOWNSHEND JRG \& SOHLBERG R. 2000. Global land cover classification at $1 \mathrm{~km}$ spatial resolution using a classification tree approach. International Journal of Remote Sensing, 21(6,7): 1331-1364.

HANSEN MC, DEFRIES RS, TOWNSHEND JRG, SOHLBERG R, DIMICELI $C$ \& CARROLL M. 2002. Towards an operational MODIS continuous field of percent tree cover algorithm: examples using AVHRR and MODIS data. Remote Sensing of Environment, 83: 303-319.

HANSEN MC, DEFRIES RS, TOWNSHEND JRG, CARROLL M, DIMICELI $C \&$ SOHLBERG RA. 2003. Global percent tree cover at a spatial resolution of 500 meters: first results of the MODIS vegetation continuous fields algorithm. Earth Interactions, 7: 1-15.

JUSTICE CO, TOWNSHEND JRG, VERMOTE EF, MASUOKA E, WOLFE RE, SALEOUS N, ROY DP \& MORISETTE JT. 2002. An overview of MODIS Land data processing and product status. Remote Sensing of Environment, 83: 3-15.

KALUZNY SP, VEGA SC, CARDOSO TP \& SHELLY AA. 1998. S-PLUS SpatialStats: User's Manual for Windows and Unix. Springer-Verlag, New York. $327 \mathrm{pp}$.

LOVELAND TR \& BELWARD AS. 1997. The IGBP-DIS global $1 \mathrm{~km}$ land cover data set, DISCover: first results. International Journal of Remote Sensing, 18: 3289-3295.

MOREIRA AAN \& LIMA GR. 1977. Relevo. In: GALVÃO MV. (Coord.). Geografia do Brasil: Região Sul. Rio de Janeiro: Fundação Instituto Brasileiro de Geografia e Estatística (IBGE). p. 1-34.

NIMER E. 1977. Clima. In: GALVÃO MV. (Coord). Geografia do Brasil: Região Centro-Oeste. Rio de Janeiro: Fundação Instituto Brasileiro de Geografia e Estatística (IBGE). p. 35-79.
PARADELLA WR, SILVA MFF \& ROSA NA. 1994. A geobotanical approach to the tropical rain forest environment of the Carajás Mineral Province (Amazon Region, Brazil), based on digital TM-Landsat and DEM data. International Journal of Remote Sensing, 15(8): 1633-1648.

PARADELLA WR, BIGNELLI PA, VENEZIANI P \& PIETSCH RW. 1997. Airborne and spaceborne Synthetic Aperture Radar (SAR) integration with Landsat TM and gamma ray spectrometry for geological mapping in a tropical rainforest environment, the Carajás Mineral province, Brazil. International Journal of Remote Sensing, 18(7): 1483-1501.

PARADELLA WR, SANTOS AR, DALL'AGNOL R \& PIETSCH RW. 1998. A Geological Investigation Based on Airborne (SAREX) and Spaceborne (RADARSAT-1) SAR Integrated Products in the Central Serra dos Carajás Granite Area, Brazil. Canadian Journal of Remote Sensing, 21(4): 376-392.

PRODES. Projeto de Estimativa do Desflorestamento Bruto da Amazônia. 2002. Disponível em: <http://www.obt.inpe.br/prodes/index.html>. Acesso em: 20 dez. 2002.

QUINLAN JR. 1986. Induction of decision trees. Machine Learning, 1(1): 81-106.

QUINLAN JR. 1993. C4.5: programs for Machine learning. San Mateo, CA: Morgan Kaufmann Publishers. 302 pp.

REED BD, BROWN JF, VANDERZEE D, LOVELAND TR, MERCHANT JW \& OHLEN D0. 1994. Measuring phenological variability from satellite imagery. Journal of Vegetation Science, 5: 703-714.

SALOMONSON VV \& TOLL DL. 1990. Execution phase (C/D) spectral band characteristics of the EOS Moderate Resolution Imaging Spectrometer (MODIS-N) facility instrument. Advances in Space Research, 2(3): 231-236.

SHIMABUKURO YE \& ALMEIDA FILHO R. 2002. Processamento digital de imagens multitemporais Landsat-5 TM e JERS-1 SAR aplicado ao mapeamento e monitoramento de áreas de alteração antrópica na Amazônia. Geografia, 27(2): 81-96.

STRAHLER A, MUCHONEY D, BORAK J, FRIEDL M, GOPAL S, LAMBIN E \& MOODY A. 1999. MODIS Land Cover and Land-Cover Change products algorithm theoretical basis document (ATBD). Version 5.0. Center for Remote Sensing, Department of Geography, Boston University, May, $72 \mathrm{pp}$.

VENABLES WW \& RIPLEY BD. 1994. Modern applied statistics with S-PLUS. New York: Springer-Verlag, 548 pp. 


\section{NOTAS SOBRE OS AUTORES}

Marcelo Lopes Latorre. Recebeu o título Oficial Aviador pela Academia da Força Aérea em 1988, e de mestre e doutor em Sensoriamento Remoto pelo Instituto Nacional de Pesquisas Espaciais (INPE) em 1998 e 2005, respectivamente. Trabalhou como Pesquisador do Instituto de Estudos Avançados (IEAV) entre 1995 e 2004. Atualmente, trabalha no Comando-Geral de Tecnologia Aeroespacial (CTA), tendo como uma das funções o controle e acompanhamento dos diversos projetos deste Comando.

Osmar Abílio de Carvalho Júnior. Recebeu o título de geólogo pela Universidade de Brasília em 1990, e de mestre e doutor em prospecção mineral pela Universidade de Brasília em 1995 e 2000, respectivamente. Trabalhou como Pesquisador do Instituto Nacional de Pesquisas Espaciais (INPE) entre 2002 e 2004. Atualmente é professor da Universidade de Brasília e bolsista de produtividade e pesquisa do CNPq onde realiza pesquisas sobre o processamento digital de imagens multiespectrais e hiperespectrais.

João Roberto dos Santos. Engenheiro Florestal pela Universidade Federal Rural do Rio de Janeiro - UFRRJ, em 1974, com mestrado em Sensoriamento Remoto pelo Instituto Nacional de Pesquisas Espaciais (INPE) em 1979 e título de Doutor em Ciências Florestais pela Universidade Federal do Paraná - UFPR em 1988. Pesquisador Titular da Divisão de Sensoriamento Remoto (DSR/INPE), atuando no Grupo de Pesquisa em Ecossistemas Terrestres, é consultor ad hoc do CNPq, CAPES, FAPESP, Fundação Araucária, EMBRAPA, FAPEAM

Yosio Edemir Shimabukuro. Recebeu o título de Engenheiro Florestal pela Universidade Federal Rural do Rio de Janeiro em 1972, de mestre em Sensoriamento Remoto pelo Instituto Nacional de Pesquisas Espaciais (INPE) em 1977, de PhD em Sensoriamento Remoto/Ciências Florestais pela Colorado State University (Estados Unidos) em 1987, e Pós-doc em Sensoriamento Remoto pela NASA/GSFC no período de 1992 a 1994. Atualmente é Pesquisador Titular da Divisão de Sensoriamento Remoto (DSR) do INPE e bolsista de produtividade e pesquisa do CNPq. 